\title{
Investigation of Electrical Interference towards Phosphene-Based Walking Support System
}

\author{
Manami, $\mathbf{K}^{1}$, Tan Xuan, $\mathbf{P}^{2}$, Eiji, $\mathbf{K}^{3}$ \\ ${ }^{1,2,3}$ Shibaura Institute of Technology, 3-7-5 Toyosu, Koto-ku, Tokyo, JAPAN \\ nb18104@shibaura-it.ac.jp
}

Article History: Received: 10 November 2020; Revised: 12 January 2021; Accepted: 27 January 2021; Published online: 05 April 2021

\begin{abstract}
A walking support system with phosphenes for blind people has been investigated. Phosphene is a phenomenon where a flash of light is recognized in the brain by giving an electrical stimulus to human's visual pathway. Phosphenes can be perceived even if their eyes are closed or they are blind. It has been clarified that phosphenes can be induced to several directions if electrodes placements are precisely selected. When phosphenes are presented to two directions for recognizing two obstacles, two pairs of electrodes must be applied. In such a case, however, the electrical interference occurs due to the short distance between electrodes. In the practical use of the phosphene-based walking support system, the avoidance of electrical interference is significant in order to present the phosphenes precisely. Therefore, in this paper, we first practically investigate the electrical interference by considering the difference in phosphene induction generated by a single pair of electrodes and by two pairs of electrodes. Then, the solutions to avoid the electrical interference are discussed.
\end{abstract}

Keywords:walking support system, blind people, phosphene, electric interference

\section{Introduction}

When blind people are going out, they usually use a white stick which is a traditional walking support tool. They hold the white stick in a hand and recognize obstacles around them by feeling something touch the stick. However, they cannot use both hands even in an emergency situation since they are using a hand for holding the white stick. Therefore, hands-free walking support systems for blind people have been increasingly paid attention to by both academia and industry. Sonic Guide is one of the hands-free walking support systems using three ultrasonic sensors which measure the distance to an obstacle from the user Tadayuki (1993). Then, the distance is notified to the user by sound using different frequencies. However, such a notification sound can interfere with other sounds, resulting in making the user act incorrectly. Meanwhile, Navi Hat Miyuki et al. (2015) leverages three ultrasonic sensors to detect the direction of an obstacle and three vibration motors to notify the user of it. These devices are installed inside a hat and the user can recognize the direction of the obstacle, namely, left, center, and right. However, it cannot detect obstacles located in diagonal directions, hence, it is of no practical use as a walking support system.

It is important that walking support systems intuitively support blind people without blocking other sensations. Hence, a new walking support system using visual information has been investigated in our recent research. This system uses electrical phosphenes. Phosphene is a phenomenon where a flash of light is recognized as visual information in the brain by giving an electrical stimulus to human's visual pathway. One of our previous studies Manami et al. (2016) clarified that the position of perceived phosphenes can be controlled by changing the electrodes placements. Therefore, the blind people can recognize the direction of an obstacle by indicating the phosphene, e.g. on the right, in the center, and on the left. However, it was difficult to induce phosphenes in the center with a single pair of electrodes placement Manami et al. (2016). Furthermore, the improvement of the accuracy of perceived phosphene positions in the center direction using two pairs of electrodes was discussed Manami et al. (2018a). When the two pairs of electrodes were used to induce phosphenes, the electrical interference must have been taken into consideration. To notify the user of the positions of two obstacles, at least two directions as perceived phosphene positions must be covered without electrical interference. However, there are no studies which clarify how to control the electrical interference with two pairs of electrodes and how to avoid the interference. These concerns should be studied for the practical use of a phosphene-based walking support system.

Therefore, this paper will discuss the electrical interference caused by two pairs of electrodes and the methods to avoid the interference towards the practical use of the phosphene-based walking support system. The rest of paper is organized as follows: In section 2, the related work is stated and section 3 clarifies the influence of electrical interference on perceived phosphene positions as well as a verification experiment. The experimental results are discussed in section 4 and finally, section 5 concludes this paper. 


\section{Related Work}

Phosphene is a phenomenon where a flash of light is recognized as visual information in the brain by giving an electrical stimulus to human's visual pathway. tACS (transcranial Alternating Current Stimulation) is one of the noninvasive methods to stimulate the human body. When the electrodes are attached to the skin, the body around them are stimulated. Although tACS does not cause serious side effects Brunoni et al. (2011), the stimulation intensity should be adjusted considering the user's sensation. The phosphene mechanism has been investigated toward the practical use of walking support systems for blind people. Laakso et al. have investigated the electrical current path around eyeballs by computer simulation when tACS stimulates human brain Laakso et al. (2013). In their simulation, the electrical current flows to a visual retina directly along the eye orbit, meaning that the electrical current at the temporal side of eyeball stimulates the temporal visual retina. However, the result of our previous work Manami et al. (2016) indicated the contradiction to their simulation result. The anatomical relation between visual retinae and human vision is depicted in Figure 1. The crossed visual information is projected onto retinae and the central area in human vision is processed around fovea in both eyeballs Teresa (2017). Even though the temporal retina processes the left side of human vision and the nasal retina processes the right side of human vision in the right eyeball as shown in Figure 1, the phosphene was observed at the right side of human vision when the skin around the temporal side of the right eyeball was stimulated Manami et al. (2016). On the other hand, one of our previous studies reported that the electrical current does not seem to flow to a retina directly along the eye orbit according to the result of a real experiment, where the positional relation between the electrodes and the visual retina was changed by eye movement Manami et al. (2018b). The previous work Manami et al. (2018b) mentioned a hypothesis about phosphene induction mechanism, that is, the phosphene is induced by voltage shift inside the eyeball, where the area of retina located at the opposite side of the eyeball from the electrodes is evoked. Therefore, when two pairs of electrodes are placed on human's face, the voltage shift by each electrodes interferes with each other. This interference must have a great impact on perceived phosphene positions. It is important to precisely control the perceived phosphene positions for walking support systems, hence, the electrical interference by two pairs of electrodes should be investigated.

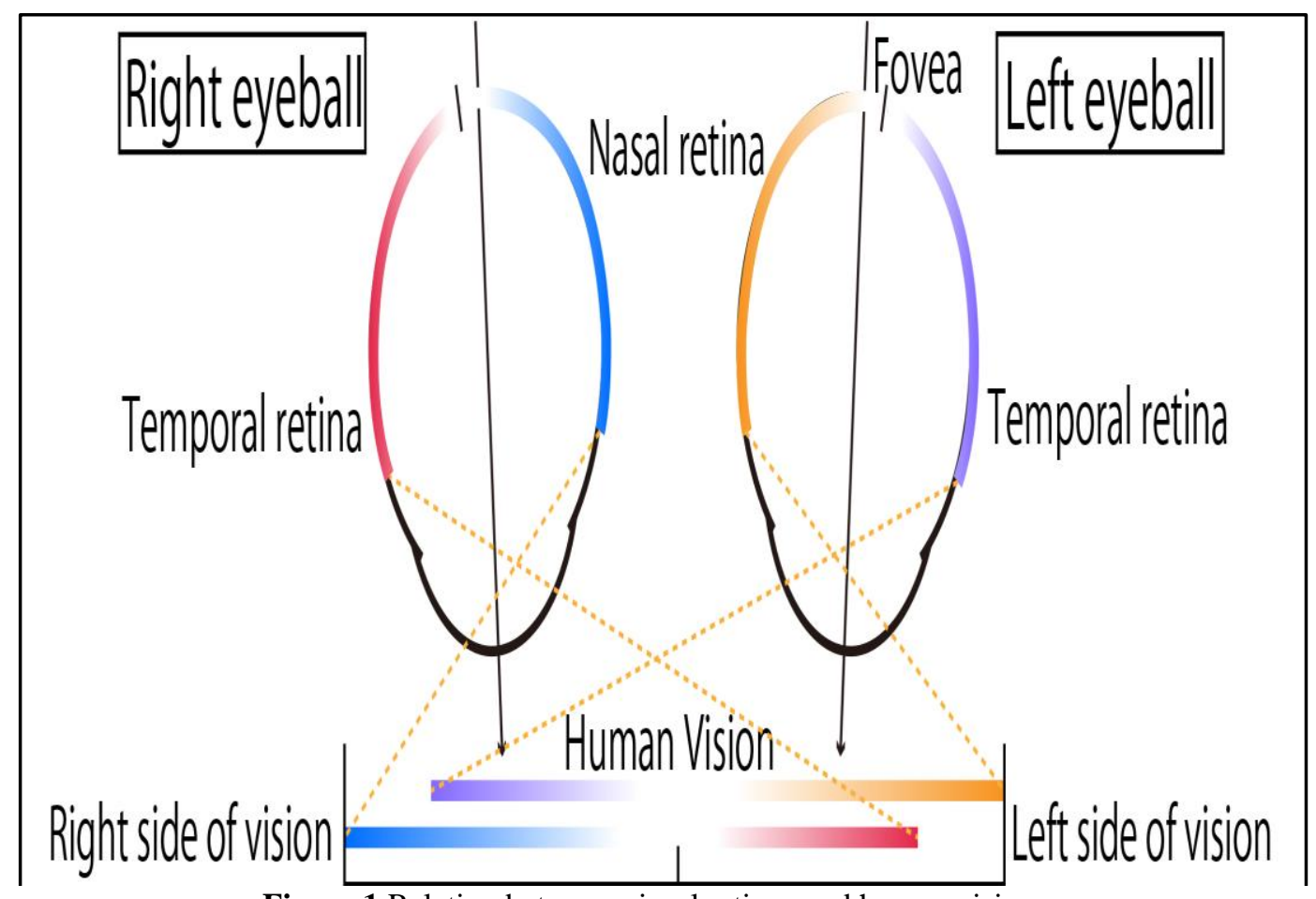

Figure 1.Relation between visual retinae and human vision 


\section{Influence of Electrical Interference on Perceived Phosphene Positions}

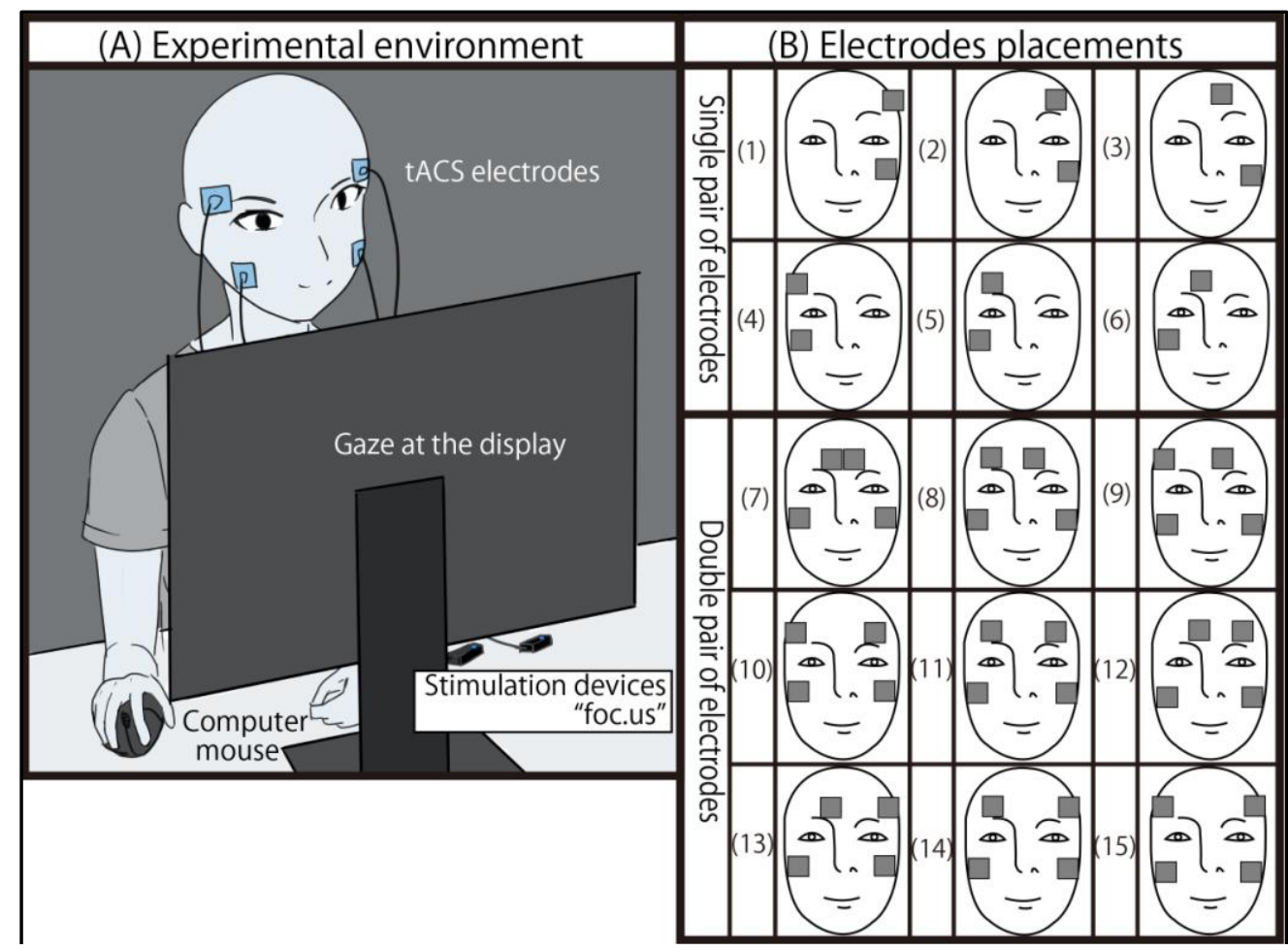

Figure 2.Experimental conditions using a single and two pairs of electrodes

As mentioned in the previous section, it can be assumed that when two pairs of electrodes are used, the perceived phosphene positions are different from the ones using a single pair of electrodes. In this paper, the influence of electrical interference using two pairs of electrodes with the short distance is clarified. Therefore, this section discusses how the perceived phosphene positions vary when the distance of each pair of electrodes is gradually changed through an experiment.

Figure 2(A) shows the experimental environment. Each subject was asked to sit on the chair in a dark room and gaze at the center of the computer display. Since this study has been conducted focusing on hands-free walking support systems, the stimulation device utilized in the experiment must be small and lightweight. For such a reason, foc.us Ienca et al. (2018) was selected as a suitable tACS device. Two pairs of electrodes placements used in this experiment are shown in Figure 2(B). In this experiment, the electrical current of 0.3 or $0.8[\mathrm{~mA}]$ were selected depending on the subject's sensation, following the previous work Manami et al. (2018a), whereas, the voltage and frequency were set to constant values of 10 [V] and 10 [Hz], respectively. As the subjects, twenty healthy people participated in this experiment. They were asked to draw the positions of perceived phosphenes as ovals on a Microsoft Paint plain page using a computer mouse. For the evaluation, images drawn by all the subjects with the same stimulation were overlaid onto an image by applying a moving average in order to get the tendency of the perceived phosphene positions. Moreover, each image was normalized based on the number of perceived phosphenes to compare the characteristics on the same scale.

Figure 3 shows the experimental result varying the electrodes placements from (1) to (15). From (1) to (6), a single pair of electrodes was used. On the other hand, from (7) to (15), two pairs of electrodes were used. The higher degree of redness the area has, the more frequently the phosphenes were perceived, whereas the higher degree of blueness the area has, the less frequently the phosphenes were perceived. The cross marks indicate the positions where the phosphenes were perceived most in the area. Since all the images depicted in Figure 3 were drawn by the subjects and overlaid, the right side of images corresponds to the right side of their vision, and the left side of images corresponds to the left side of their vision. From the result of electrodes placements (1) to (6), it seems that the phosphenes were perceived on the same side of the electrodes placed on the face. When two pairs of electrodes were used (e.g. (7)), perceived phosphene positions were different from the ones, which were made by combining two results obtained by each single pair of electrodes (e.g. (3) and (6)). This is because the electrical current from two pairs of electrodes interfere with each other, then, other areas on retinae were revoked. These results caused by electrical interference are discussed in the next section. 


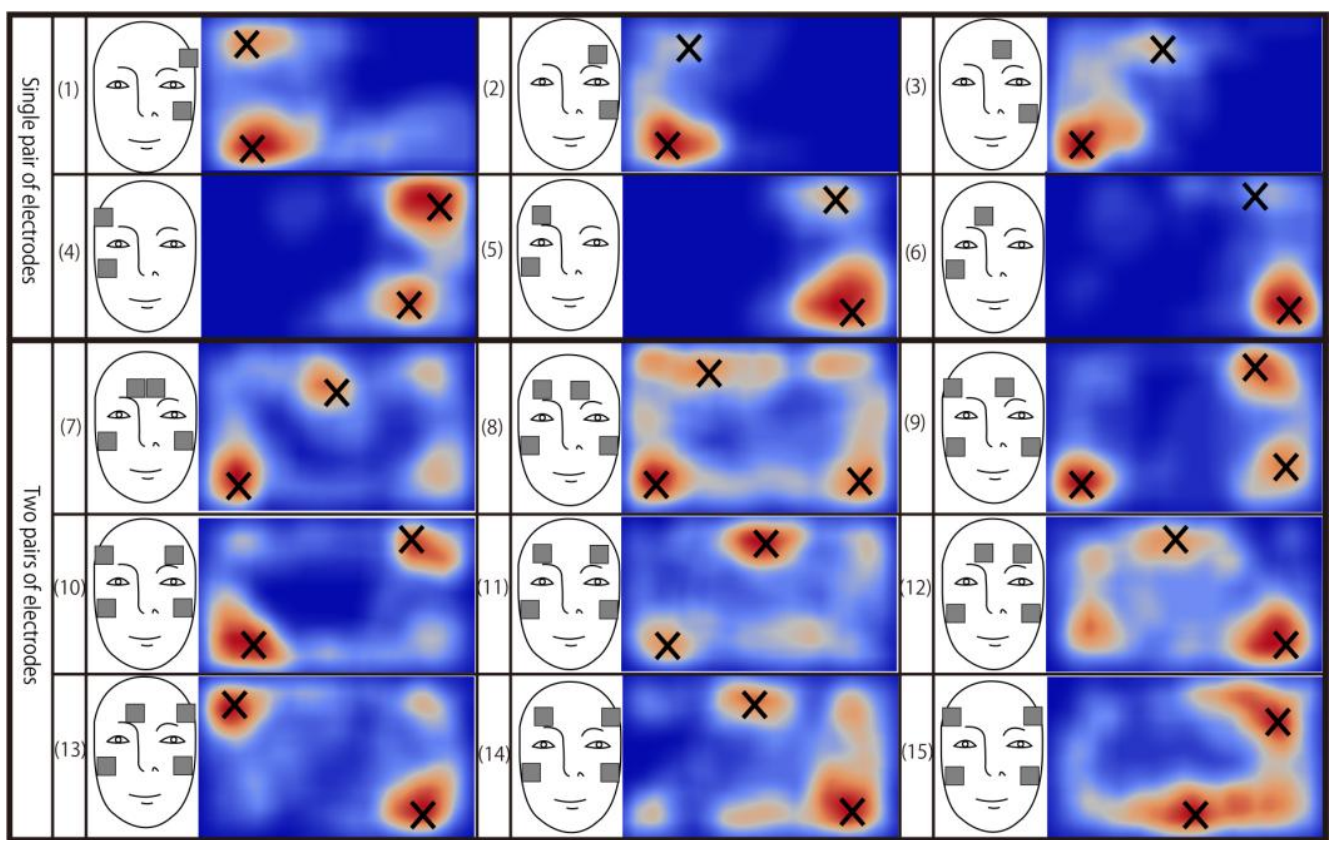

Figure 3.Perceived phosphene positions with each electrodes placements

\section{Discussion}

In this section, influence of the electrical interference on perceived phosphene positions is discussed. If there is no electrical interference by two pairs of electrodes, the perceived phosphene positions are the same as the ones made by combining two results obtained by each single pair of electrodes. If there are any differences between these two, they can be regarded as the influence of the electrical interference and the characteristics can also be clarified.

Expected evoked retinal areas based on the hypothesis in the previous work Manami et al. (2018b) are introduced here in order to investigate the positional relation with the electrodes. Column (i) in Figure 4 shows top views of eyeballs. Gray squares indicate the positions of two pairs of electrodes and cross marks indicate the evoked retinal areas. Column (ii) in Figure 4 shows perceived phosphenes in each electrodes placements from (7) to (15), which are the same as the ones in Figure 2. Column (iii) in Figure 3 shows images of perceived phosphenes which were made by combining two results obtained by the corresponding two single pairs of electrodes. Column (iv) in Figure 4 shows the images obtained by subtracting the values of image data in column (iii) from the ones in column (ii). Note that normalization was performed to images in column (iii) and in column (iv) to clarify the characteristics. In images in column (iv) in Figure 4, the higher degree of redness the area has, the more enhanced phosphenes were perceived, whereas the higher degree of blueness the area has, the more weakened phosphenes were perceived. From the images in column (iv) in Figure 4, four types of characteristics can be clarified as follows:

First, when two pairs of electrodes were placed with a small distance such as electrodes placements of (7), (8), and (12), more phosphenes were perceived in the center of human vision compared to the images in column (iii). On the other hand, in the electrodes placements of (9) and (13), the enhanced perceived phosphenes were not so much in the center. This is because the electrical interference is not very strong with these electrodes placements.

Second, when several phosphenes were induced in the cases of (11), (12) and (14), the perceived phosphene positions were completely different from the ones expected from the evoked retinal areas. This is because there is a tendency that perceived phosphenes can be merged due to the effect of light cognition. When several phosphenes are induced nearby with high brightness, they cannot be differentiated in human vision. This is related to the detection accuracy of obstacles for a walking support system. If a high spatial resolution is required, the given electrical stimulus should not be very strong so that the user can recognize several phosphenes separately with low brightness. In the future, this adjustment of stimulus degree can be discussed based on the hypothesis that the phosphene induction mechanism has a connection with the electrical potential of eyeballs Manami et al. (2018b). 
Third, when phosphenes were induced in the lower part of human vision, there is a tendency that the phosphenes were not perceived well. This tendency can be seen in the most of electrode placements except (8) and (9). This can be referred to the location of each electrode. A pair of electrodes is composed of two electrodes, of course. In this experiment, one is placed just above an eyeball and the other is placed on the cheek. In such a case, induced phosphenes in the upper part are bright and the ones in the lower part are dark. This is because the electrode on the cheek is far from the eyeball, hence, the electrical potential at the lower part is low. This problem can be solved by moving the electrode location on the cheek to upper area near the eyeball, referring to the presumption stated in Manami et al. (2018b).

Fourth, when two pairs of electrodes were placed with the electrodes placements of (9), (10), and (15), perceived phosphenes on the right side were enhanced and the ones on the left side were weakened. This is because of the difference in the stimulation timing. In this experiment, two stimulation devices were used to give electrical stimulus to two locations, and these two devices were controlled independently. Therefore, the stimulation timing to the two locations were not completely the same, thus, the perceptions of phosphenes on the right and left sides were different. This problem can also be solved by automatically synchronizing the control of two devices when this is used as a walking support system.

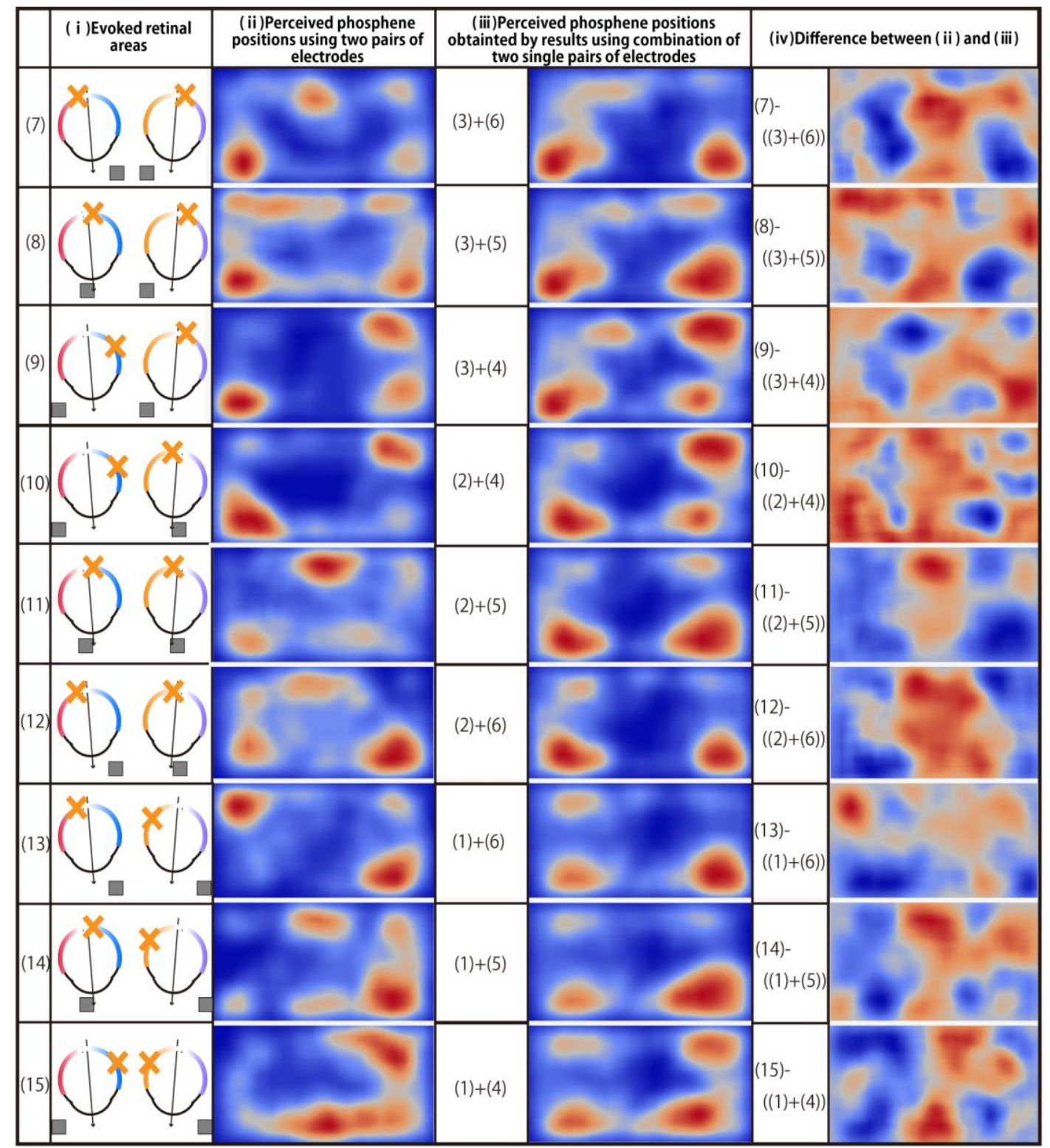

Figure 4.Difference of perceived phosphene positions between using two pairs of electrodes and using combination of two single pairs of electrodes 


\section{Conclusion}

In this paper, the electrical interference by two pairs of electrodes and the methods to avoid the interference were discussed. It was revealed that there are four types of characteristics in perceived phosphene positions when two pairs of electrodes are used. First, when the distance between two pairs of electrodes is short, a simple electrical interference is observed. Second, when several phosphenes are induced nearby, there is a tendency that the perceived phosphenes are merged due to the effect of light cognition. Third, when one of the electrode is placed on the cheek, the phosphenes induced in the lower part cannot be perceived well due to the distance to the eyeball. Fourth, phosphenes cannot be perceived well when two pairs of electrodes are controlled independently, resulting in different stimulation timing. It is concluded that the adjustments of electrodes placements and the degree of electrical stimulus, and the mechanism of automatic synchronized control of two stimulating devices are needed in order to avoid the electrical interference stated above. However, those ideas have not as yet been realized or even been verified. In the future, therefore, it is required to verify these hypotheses through real experiments to practically implement them in a walking support system.

\section{References}

1. Brunoni A.R., Amadera J., Berbel B., Volz M.S., Rizzerio B.G. and Fregni F. 2011. A systematic review on reporting and assessment of adverse effects associated with transcranial direct current stimulation. Int J Neuropsychopharmacol., 14(8): 1133-1145.

2. Laakso and A. Hirata. 2013. Computational analysis shows why transcranial alternating current stimulation induces retinal phosphenes. J Neural Eng., 10(4): 1-9.

3. Ienca M., Haselager P. and Emanuel. E. 2018. Brain leaks and consumer neurotechnology. Nat Biotechnol., 36: 805-810.

4. Manami K. and Eiji K. 2016. Walking Support System for Blind People using Phosphene. Proceedings of the 10th South East Asia Technical University Consortium Symposium (SEATUC2016)., CD-ROM.

5. Manami K. and Eiji K. 2018a. Spatial Relation between Given Stimulus and Perceived Phosphene. Proceedings of the 12th South East Asia Technical University Consortium Symposium (SEATUC2018)., CD-ROM.

6. Manami K., Yuhi K., Yoshihiro N., P. X. Tan and Eiji K. 2018b. Study on Relation between Stimulated Parts of Retina and Perceived Positions of Phosphene towards Realization of Walking Support System for Blind People. Proceedings of IEICE Society Conference., 118(305): 25-30 (in Japanese).

7. Miyuki S. and Eiji K. 2015. Navi Hat: Collision Avoidance Wearable Device for Blind People. Graduation Thesis. Shibaura Institute of Technology (in Japanese).

8. Tadayuki S. 1993. Electronics for Walking Support for Blind People. BME., 7(7): 15-20 (in Japanese).

9. Teresa S. 2017. Neuroscience: Exploring the Brain fourth edition. LIPPINCOTT. 